\title{
The Logic of Exempting CRISPR/Cas9-Plants from Strict GM Approval under European Union GM Regu- lation: A Case Study of Genome Editing in Camelina
}

Jarst van Belle, Jan Schaart, Robert van Loo*

\section{Introduction}

Genome editing - by which the genetic makeup of an organism can be altered with high precision - has become a hot topic in plant breeding, particularly the use of CRISPR/Cas9. CRISPR/Cas9 technology emerged only 4 years ago, and is increasingly being used in research. ${ }^{2}$ CRISPR/Cas9 also plays a key role in the Horizon 2020 EU project, COSMOS (Camelina \& crambe Oil crops as Sources for Medium-chain Oils for Specialty oleochemicals), which aims to improve the camelina oil crop (Camelina sativa). Camelina has an interesting oil profile thanks to its high levels of typical monounsaturated fatty acids (MUFAs). ${ }^{3}$ Using CRISPR/Cas9 genome editing, the camelina oil profile can be further improved for use in the chemical industry (see Section III). Further metabolism of MUFAs to undesired PUFAs (polyunsaturated fatty acids) - which occurs in wild type plants can be prevented, leading to higher end levels of MUFAs. ${ }^{4}$ These MUFAs can be chemically converted into medium-chain fatty acids (MCFAs) and could replace MCFAs from palm kernel and coconut oils, several hundred thousand metric tons of which are imported into the EU-28 each year. ${ }^{5} \mathrm{Im}$ proved camelina cultivars could therefore reduce the EU's dependency on imported palm oil.

CRISPR/Cas9 technology, which will be used to produce targeted mutations in specific genes, is included in COSMOS for both technical and economic reasons. Camelina is a polyploid crop and has six copies of each gene. ${ }^{6}$ In order to meet the COSMOS breeding targets for camelina, at least

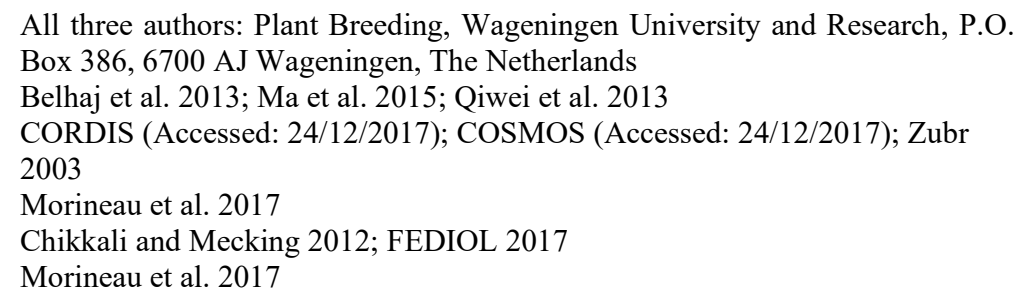


six independent mutation events are required in the target gene. Inducing multiple mutations using conventional breeding methods (see Section II), in which random mutations are induced, is difficult, laborious, and timeconsuming. ${ }^{7}$ With CRISPR/Cas9, however, multiple targeted genome edits can be achieved in a single step. ${ }^{8}$ CRISPR/Cas9, therefore, has the technical advantage of targeted induced mutations, which can save time and money, especially when working with polyploid crops.

The inclusion of CRISPR/Cas9 in COSMOS also has political consequences. COSMOS aims to develop the new camelina variety for the EU market, which, under the current legislation, does not favor GMOs (other than those created through 'traditional' mutation breeding). ${ }^{9}$ Yet CRISPR/Cas9-products are still regarded as GMOs in the EU, and fall under EU GM regulation, which is based on the Precautionary Principle (see Section IV). However, CRISPR/Cas9-plants can be produced without the permanent presence of foreign DNA (i.e., DNA harboring the CRISPR/Cas9 machinery) in its genome. This makes CRISPR/Cas9-products indistinguishable from conventional equivalents on a genetic and phenotypic level.

Despite the innovative advantages of CRISPR/Cas9, there is currently a great debate as to whether genome edited plants should be classified as GMOs. However, there does not seem to be a technical reason to treat these plants as different than conventionally bred plants. Therefore, there is an urgent need for the EU to change the way it regulates CRISPR/Cas9, and exempt genome edited products from the strict approval procedures of GM regulation.

This article describes and compares different plant breeding methods from a technical perspective (Section II). The importance and innovation of genome editing in plant breeding is illustrated using camelina as a case study (Section III). The current EU approach to the regulation of GM is also compared with another existing legal framework (Section IV). Using a scientific perspective, a case is made for exempting genome edited products from the strict approval procedures involved in current EU GM regulation.

Yang, Wang and Zhao 2017

Ma et al. 2015; Morineau et al. 2017

Hartung and Schiemann 2014; Jones 2015; Merchant and Stevens 2017 
Van Belle et. al.

\section{Genome Editing: The Next Step in Plant Breeding}

Conventional breeding includes classical breeding and 'traditional' mutation breeding. Classical breeding aims to domesticate and further improve the characteristics of organisms (e.g., yield, nutritional value, and (a)biotic stress tolerance and resistance) by deliberately crossing within a species or between breed-compatible related species. Together, breed-compatible species form a gene pool that includes all the naturally available genetic variation which can be exploited by classical breeding. In other words, classical breeding is limited to naturally-existing genetic variation. 'Traditional' mutation breeding - which emerged in the twentieth century - increases genetic diversity using ionizing radiation (X-rays, gamma rays) and chemical mutagens such as EMS (ethyl methyl sulfonate). It induces the same type of DNA mutations that occur in nature, but does so at a higher rate. ${ }^{10}$

Genetic modification involves the direct manipulation of an organism's genome (i.e., gene transfer or gene mutation), and includes cisgenesis and transgenesis (which involve gene transfer) and mutation breeding (which involves gene mutation). It includes both novel genome editing and 'traditional' mutation breeding. Even though 'traditional' mutation breeding is a form of genetic modification, it can be considered a form of conventional breeding, ${ }^{11}$ and has a 'history of safe use' (it has been in use for decades, and a significant proportion of the food we eat is the product of conventional mutagenesis, with no evidence of harm). Mutants created via 'traditional' mutation breeding are, therefore, exempt from the strict approval procedures of GM regulation.

Genome editing, a novel form of genetic modification and mutation breeding, involves precise alterations of the genetic makeup of an organism (i.e., mutating an exact location in the genome). ${ }^{12}$ This precision distinguishes genome editing from other forms of genetic modification such as cis- and transgenesis (Fig. 1; i.e. the insertion and random integration of genetic material into an organism of interest, by which the DNA originates from a breed-compatible and breed-incompatible species, resp.), and from other forms of mutation breeding, in which multiple random mutations 'traditionally' alter the genetic makeup of an organism. The random induction of mutations means that thousands of individuals have to be screened. ${ }^{13}$ For example, the commonly-used mutagenic chemical EMS causes the random

10 Adams and Wendel 2005; Camara, Ancell and Pigliucci 2000

11 Constable 2007

12 Belhaj et al. 2013; Ma et al. 2015; Qiwei et al. 2013

13 Yang, Wang and Zhao 2017 
transition of GC base pairs into AT base pairs, and thus the substitution of DNA building blocks. ${ }^{14}$ Because relatively low mutation frequencies must be achieved (if the frequency is too high, it is lethal), many thousands of mutated plants need to be produced and screened to find a mutation in the target gene. This mutation should not only be located at the desired target, but should also be effective, as only a limited number of substitution mutations leads to altered gene activity.

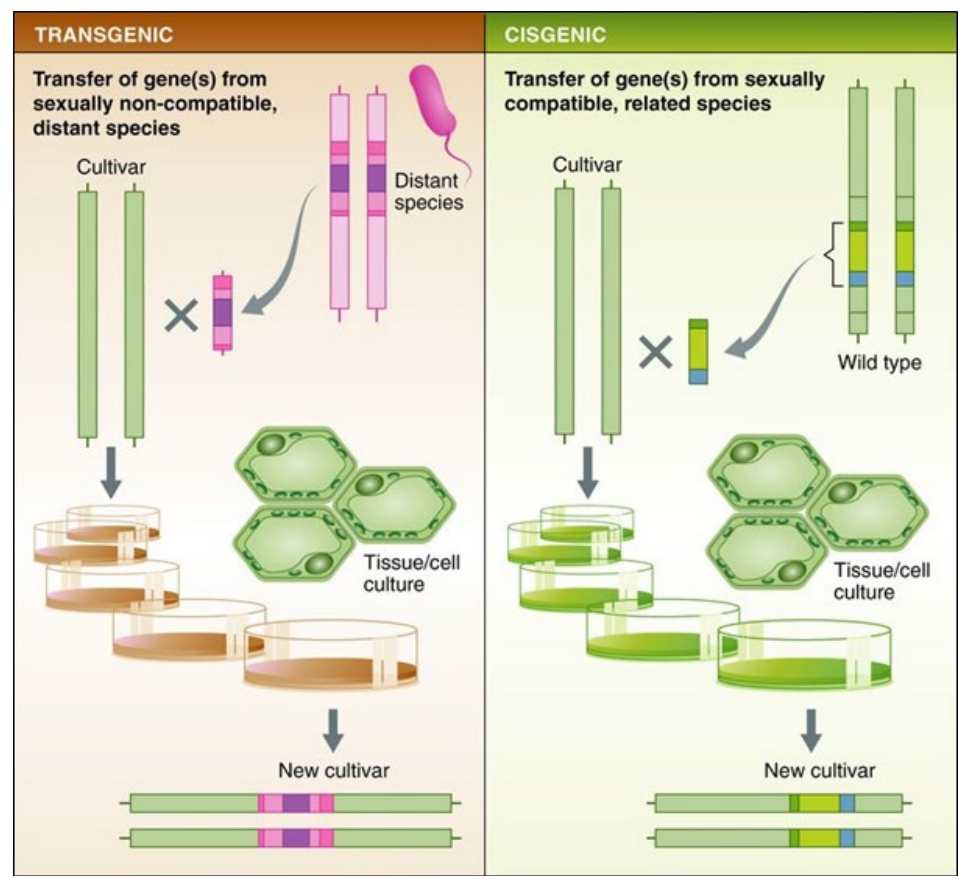

Figure 1: An illustration of transgenic and cisgenic genetic modification of plants, adapted from Hunter 2014. ${ }^{15}$ Transgenesis includes the transfer of genetic material from a non-compatible species (either another plant or, for example, a bacteria, as shown in the figure) to the plant of interest, while cisgenesis includes transfer from a compatible species. Unlike genome editing (see Fig. 2), transgenesis and cisgenesis require the insertion of extra DNA into the organism of interest, and the location of the insertion is a random process. Tissue and cell culture technology is commonly used to transform and regenerate plants as shown in the figure. Eventually a new genetically engineered cultivar with either transgenic or cisgenic material can be developed.

Unlike 'traditional' mutation breeding, genome editing relies on targeted mutations rather than accidental desired mutations among a relatively high

$14 \quad$ Lightner and Caspar 1998

15 Hunter 2014 
number of off-target mutations (e.g. in rice, 1 out of 16 to 2800 EMS mutations is located in the desired gene). ${ }^{16}$ Targeted mutation breeding using genome editing has a clear advantage over 'traditional' mutation breeding, in that desired mutations are much easier to achieve, particularly combined mutations by, for example, targeting all copies of a gene of interest in a single plant. And unlike with 'traditional' mutation breeding, screening many thousands of plants is no longer necessary when using genome editing technology.

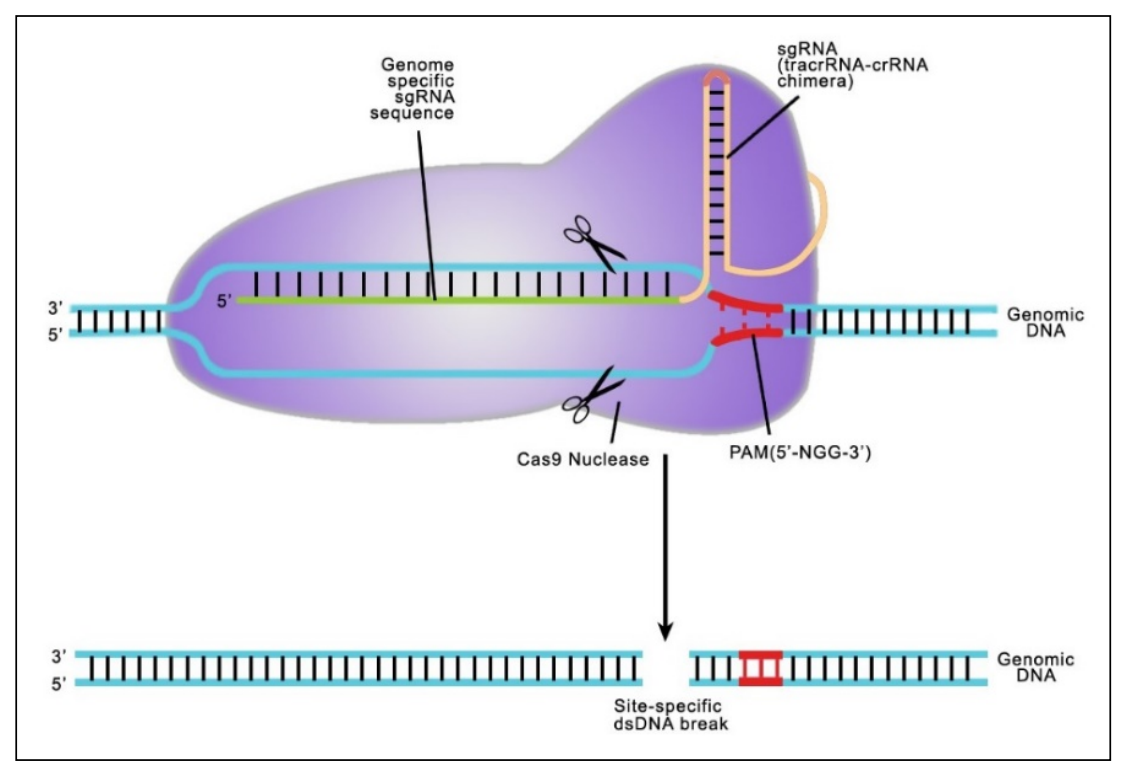

Figure 2: The molecular mechanism of CRISPR/Cas9 genome editing, from GeneCopoeia. ${ }^{17}$ To induce mutations, the CRISPR/Cas9 tool uses an RNA sequence specifically designed to recognise and anneal to the target DNA, and a Cas9 protein to create a double-strand DNA break. The natural repair mechanism of the DNA (not shown in the figure) is error-prone, often resulting in deletions and subsequently disrupted genes, this being one of the applications of CRISPR/Cas9 genome editing. Because CRISPR/Cas9 activity depends on the recognition of DNA by the RNA, the induced mutations are rather specific (cf. radiation- and chemical-induced mutations that are used in 'traditional' mutation breeding).

16 Maghuly and Laimer 2017; Nelson et al. 2004

17 GeneCopoeia (Accessed: 30/8/2017) 
Furthermore, compared to cis- and transgenesis, genome editing does not require the permanent insertion and integration of foreign DNA in the ultimate modified plant. ${ }^{18}$ Even if foreign DNA has been present at an early stage it can be removed by crossing at a later stage, yet the intended mutation can still be retained. ${ }^{19}$ This is possible since the foreign DNA (coding for the CRISPR/Cas9 machinery) is not, in fact, the mutation itself, but rather encodes the genome editing mechanism. The genome editing mechanism is illustrated with the CRISPR/Cas9 system in Fig. 2.

Another advantage that genome editing offers over any other form of plant breeding is the ability to tackle more complex objectives such as the simultaneous induction of multiple mutations. ${ }^{20}$ In plant breeding, multiple knockouts (i.e., disrupted genes) are useful for simultaneously improving different agronomic traits, as well as for improving single traits which need more than one genome edit due to the genetic complexity of the trait (multiple genes involved) or the plant (e.g. polyploid crops) in general. The next section of this article will illustrate the significant potential of genome editing.

\section{Genome Editing in Camelina}

The Horizon 2020 COSMOS EU project investigates camelina (Camelina sativa), an oilseed crop, with the aim of enhancing its oil profile and turning it into a profitable, sustainable, multipurpose European oil crop..$^{21}$ The seeds naturally contain an interesting oil profile, with $12-16 \%$ gondoic acid (C20:1). ${ }^{22}$ This particular MUFA has its chemical double bond in an unusual position, making it an alternative resource to the widely-used palm kernel and coconut oil, lauric acid (C12). ${ }^{23}$ In 2017, the EU-28 imported 669,000 metric tons of palm kernel oil for a wide variety of uses. ${ }^{24}$

The production of palm oils is controversial. The expansion of palm oil plantations generally involves the destruction of rainforest and endangers vulnerable indigenous species such as the orangutan. ${ }^{25}$ Therefore, it is important to develop a sustainable alternative, such as camelina. The unique

18 Wook Woo et al. 2015

Schiml, Fauser and Puchta 2016

Ma et al. 2015; Morineau et al. 2017

CORDIS (Accessed: 24/12/2017); COSMOS (Accessed: 24/12/2017)

Zubr 1997; Zubr 2003

Chikkali and Mecking 2003

FEDIOL 2017

Fitzherbert et al. 2008 
selling point of camelina, besides its interesting oil profile, is that this crop grows well at different European latitudes (Fig. 3) and requires a low input. ${ }^{26}$ The camelina oil profile may be further improved via breeding, meaning that novel camelina oils will be better able to compete with palm oils.

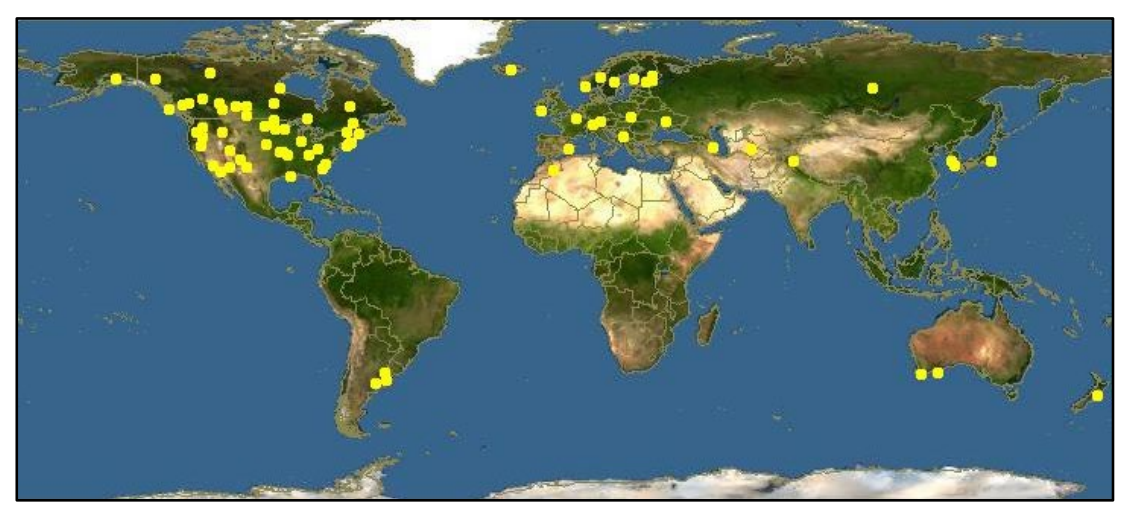

Figure 3: Countries where Camelina sativa is grown. ${ }^{27}$

The fatty acid metabolic pathway which produces gondoic acid as an intermediate product is known in camelina. Disrupting the FAD2 (fatty acid desaturase 2) gene prevents further metabolism of the desired gondoic acid and other MUFAs to undesired PUFAs (polyunsaturated fatty acids). The genetic makeup of camelina has an allohexaploid nature (Fig. $4 A$; i.e. it contains three genomes that do not recombine (exchange genetic information) with each other). ${ }^{28}$ This means that, in camelina, there are three independent genetic loci encoding FAD2 and, because genomes always exist as two copies, camelina has in total six copies of the FAD2 gene. To completely knock out FAD2 mutation by EMS is hardly feasible: firstly, three complementary effective mutations have to be induced in each FAD2 locus in separate plants, which subsequently have to be fixed by making each mutation homozygous via self-fertilization. Finally, the obtained mutants have to be crossed with each other in order to fix all mutations again in a single individual plant.

CRISPR/Cas9 genome editing can circumvent this problem by simultaneously targeting each FAD2 locus in a single plant (Fig. 4B). A complete knockout can already be obtained within two generations, without off-target

26 Agence canadienne d'inspection des aliments (Accessed: 30/8/2017); Dhuicq 2013; Zubr 1997

27 Dhuicq 2013; Discover Life (Accessed: 30/8/2017)

28 Morineau et al. 2017 
mutations (cf. EMS mutants) and without foreign DNA present in the genome. ${ }^{29}$

To summarize, genome editing offers novel possibilities for plant breeding. In particular, genome editing in camelina makes it possible to effectively improve the camelina oil profile in a short time, and to compete with palm oils, which would not only help efforts to preserve rainforests but would also boost the EU economy. This may only be one example of genome editing, but the options for plant breeding have expanded significantly. Today, only minimal changes need to be made in the genetic makeup of organisms, compared to classical breeding and other forms of genetic modification. Genome editing really is the next step in plant breeding.

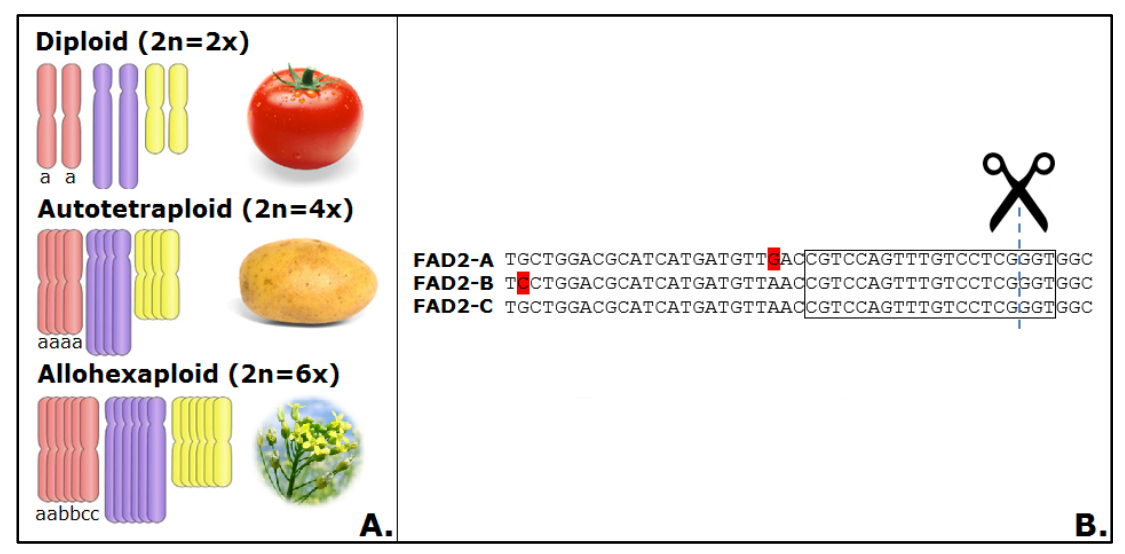

Figure 4: Relative complexity of the camelina genome and the potential of CRISPR/Cas9 genome editing in camelina. Figure 4A: Camelina is a hexaploid by nature, i.e. six copies per chromosome and generally six copies per gene (in comparison, the tomato has only two). Camelina is also an alloploid, i.e. the (six) chromosome copies belong to (three) different subgenomes (a, b, and $\mathrm{c}$ in the figure) which do not recombine (in comparison, the potato is an autotetraploid, i.e. all four chromosome copies can recombine). Figure 4B: To completely knockout the FAD2 gene in camelina in order to produce an improved oil profile, each gene copy should be mutated, thus each subgenome should be targeted. Here the red-marked nucleotides denote the genetic variation that differentiates the three different FAD2 gene copies. Using CRISPR/Cas9 genome editing (see Fig. 2 for details), a guide RNA can be designed to target a conserved DNA sequence in FAD2 (i.e. the target sequence is present in each subgenome; see the boxed area in the figure). This way, CRISPR/Cas9 is able to simultaneously induce accurate mutations in all three subgenomes. 


\section{$I V$. The Impact of Different GMO Legislations on Genome Edited Products}

Worldwide, different approaches to the governance of the industrial sector may lead to markedly different economical outcomes. This is certainly the case when it comes to current GMO legislation. Below, the EU legal framework is compared with the Canadian legal framework as an example.

In 1993, Canada established the Federal Regulatory Framework for Biotechnology, based "on principles for an efficient, effective approach for regulating biotechnology." 30 Within this framework, GM products fall under the same regulations as conventional products. This means that plants with novel traits, and novel foods, are subjected to the same risk assessments, regardless of the breeding methods used in their production. Even though GM products - including genome edited products - are always considered novel, they are subject to standard procedures before approval. ${ }^{31}$ As a result of the Canadian regulatory framework, Canada is the fourth largest producer of GMOs (other than those created from 'traditional' mutation breeding), with 13.1 million hectares in 2017, and only India close behind. ${ }^{32}$

In conclusion, Canada follows a predominantly product-based approach (i.e., it is the products, rather than the methods to obtain these products, that are reviewed) with an innovation-friendly outlook. Canada follows a precautionary approach (risk assessment applies to novel products), yet to a lesser extent than the EU. ${ }^{33}$

In 2001, the EU adopted a directive on the deliberate release of GMOs into the environment, which was followed in 2003 by a regulation on GMfood and GM-feed. ${ }^{34}$ Within this legal framework, GM products do not only fall under the same regulations as conventional products (cf. the Canadian framework): In addition, GM products (created from cis- and transgenesis and genome editing) undergo an excessively lengthy (4-6 years) and costly (7-15 million euros) process involving risk assessment, approval, labelling and traceability requirements. ${ }^{35}$ At the same time, equivalent products obtained via classical breeding are not subject to this GM regulation. Mutants

\footnotetext{
30 Law Library of Congress (Accessed: 26/8/2018); Canadian Food Inspection Agency (CFIA) 2007; MacLaughlin 2004

31 Government of Canada (Accessed: 26/8/2018)

32 Law Library of Congress (Accessed: 26/8/2018); ISAAA 2017 (1); ISAAA 2017 (2)

33 Law Library of Congress (Accessed: 26/8/2018); Montpetit 2005; Andrée 2006

34 European Parliament 2001; European Parliament 2003

35 Hartung and Schiemann 2014; Kalaitzandonakes, Alston and Bradford 2007; Merchant and Stevens 2017
} 
created via 'traditional' mutation breeding - while legally GMOs - are specifically exempted from the strict GM approval procedure. As a result of the EU regulatory framework, only one variety of a major crop (maize) from a major company (Monsanto) has currently been approved for cultivation following risk assessment. In 2017, a mere 132 thousand hectares of this maize variety were cultivated in the EU, which was only $1 \%$ of the production of GMOs in Canada, or $0.07 \%$ of the global production. ${ }^{36}$

In conclusion, the EU follows a predominantly process-based approach (i.e., the processes to obtain products are reviewed rather than the products themselves). More so than Canada, the EU follows a precautionary approach, which, in this case, is based on the Precautionary Principle: "A strategy to cope with possible risks where scientific understanding is yet incomplete, such as the risks of nanotechnology, genetically modified organisms and systemic pesticides." ${ }^{37}$ The Precautionary Principle is defined as follows:

When human activities may lead to morally unacceptable harm that is scientifically plausible but uncertain, actions shall be taken to avoid or diminish that harm. Morally unacceptable harm refers to harm to humans or the environment that is: threatening to human life or health, or serious and effectively irreversible, or inequitable to present or future generations, or imposed without adequate consideration of the human rights of those affected.

In other words, the Precautionary Principle is not applied to conventionally bred plants (conventional products only fall under standard regulations, or are specifically exempt from strict GM approval rules), while GMOs (other than those created from 'traditional' mutation breeding) may only be produced when there is absolute certainty about their safety.

While the above-mentioned regulatory frameworks were established 1525 years ago, genome editing technology only became an important topic in 2011, when Nature Methods selected genome editing as Method of the Year. ${ }^{38}$ This is of no consequence for the interpretation of the Canadian framework, as novel biotechnology products are already regulated in the same way as conventional products. In other words, no new regulations have to be created. However, the current EU framework has not yet taken

\footnotetext{
$36 \quad$ ISAAA 2017 (2)

37 World Commission on the Ethics of Scientific Knowledge and Technology (COMEST) 2005

$38 \quad$ Nature 2012
} 
genome edited plants into account and leaves room for multiple interpretations. The $2001 \mathrm{EU}$ Directive states: ${ }^{39}$

Techniques/methods of genetic modification yielding organisms to be excluded from the Directive, on the condition that they do not involve the use of recombinant nucleic acid molecules or genetically modified organisms other than those produced by one or more of the techniques/methods listed below, are: mutagenesis.

Thus, on the one hand, genome edited products such as CRISPR/Cas9 plants could fall under the GM approval rules due to the fact that they involve the integration of DNA harboring the CRISPR/Cas9 machinery into the plant's genome. On the other hand, genome edited products could be exempted from these rules on the basis that they result from mutation breeding (see Section II). Furthermore, and as mentioned before, any integrated DNA can be removed at a later stage while maintaining the intended mutation, to the point that genome edited plants are indistinguishable from conventionally bred equivalents.

Until further consensus is reached, genome edited products will remain subject to the strict GM approval procedure. Only very recently, on July $25^{\text {th }} 2018$, the EU Court of Justice made a decision about the legislation of genome edited organisms ${ }^{40}$ :

Organisms obtained by mutagenesis are GMOs and are, in principle, subject to the obligations laid down by the GMO Directive. However, organisms obtained by mutagenesis techniques which have conventionally been used in a number of applications and have a long safety record are exempt from those obligations.

In practice, this means that genome edited plants will follow the same route as other GMOs (created from cis- and transgenesis) under the EU GM regulation: the approval procedure of genome edited plants for cultivation will be too lengthy and expensive until such time as there is absolute certainty about their safety. At the same time, because no genome edited plants will be cultivated, they cannot be conventionally used in numerous applications, nor can a long safety record can be built. Consequently, genome edited plants will not be exempted from the strict GM approval procedure under the current EU GM regulation.

\footnotetext{
39 European Parliament 2001

40 Court of Justice of the European Union 2018
} 
Van Belle et. al.

\section{Discussion}

In this article, the technical aspects of different conventional and genetic modification breeding technologies have been compared, including classical breeding, 'traditional' mutation breeding, cis- and transgenesis, and genome editing - a new form of mutation breeding which uses CRISPR/Cas9 in particular. The technical bottlenecks as well as the potential of these breeding technologies have been illustrated by the genome editing of camelina, where CRISPR/Cas9 technology creates the opportunity to significantly improve the plant's oil profile. The improved oil profile of camelina, a native plant that grows well at different European latitudes and requires a low input, can directly compete with that of palm oil. As such, camelina cultivars improved by CRISPR/Cas9 technology have the potential to significantly decrease the EU's dependency on imported palm oil. This will not only benefit the EU economy, but also the rainforests that are disappearing due to the development of palm tree plantations. The efficient improvement of the camelina oil profile using CRISPR/Cas9 technology is just one of the many potential applications of genome editing: there are also novel methods to confer abiotic stress tolerance, disease resistance, and many more crop-specific traits for the development of valuable phenotypes. ${ }^{41}$

Besides comparing different plant breeding techniques and illustrating the significant potential of novel technologies, this article has also elaborated on how different government approaches to GMO legislation may impact genome edited products. As I have discussed, Canada follows a predominantly product-based approach under the Federal Regulatory Framework for Biotechnology. This framework includes both precautionary and innovation-friendly components for novel products, regardless of the breeding method used. As a result, Canada is one of the largest producers of GMOs (other than those created from 'traditional' mutation breeding), and genome edited plants are treated in the same way as any other plant with a novel trait. The EU follows a predominantly process-based approach under the 2001 Directive on the deliberate release of GMOs into the environment and the 2003 directive on GM food and GM feed. The EU GM regulation follows a precautionary approach, which is based on the Precautionary Principle. GM products - including genome edited products - must undergo extensive assessment, unlike conventional products which do not fall under

41 Feng et al. 2013; Jiang et al. 2013; Jouanin et al. 2017; Li et al. 2013; Mao et al. 2013; Miao et al. 2013; Nekrasov et al. 2013; Osakabe et al. 2016; Qiwei et al. 2013; Upadhyay et al. 2013; Xie and Yang 2013; Zhang, Wen and Guo 2014 
GM regulation or are specifically exempted from the strict GM approval procedure (i.e., products created via 'traditional' mutation breeding). As a result, only one GM variety of a major crop from a major company is cultivated in the EU following the crop's approval under GM regulation.

In other words, the legislation of - for example - genome edited camelina with an improved oil profile, which promises both economic and ecological benefits, will currently be troublesome in the EU. Meanwhile, other world economies have more freedom to develop plants using modern breeding technologies. This is not only the case for Canada, but also for, e.g., the USA, where the US Department of Agriculture confirmed on March $28^{\text {th }}$ 2018 that genome edited plants (which could otherwise be obtained through conventional breeding methods) will not be regulated. ${ }^{42}$

At the same time, the most modern breeding technology, CRISPR/Cas9, allows extremely precise altering of the genetic makeup of a plant without necessarily integrating foreign DNA. ${ }^{43}$ This would make any additional negative effects due to genome editing more predictable than those effects which result from the use of 'traditional' mutation breeding, transgenesis and cisgenesis, and even classical breeding. Because mutations induced by genome editing could also be achieved by natural events (spontaneous mutations) or 'traditional' mutation breeding, genome edited plants are no different from conventionally bred equivalents on a genetic or phenotypic level. As a consequence, genome edited plants and derivative products pose no more risks to humans or the environment than conventionally bred equivalents. By now, there is even a well-established scientific consensus that organisms produced by using modern breeding technologies are, at least in principle, safe for both humans and the environment. ${ }^{44}$ In conclusion, from a scientific perspective, there is no reason to treat genome edited plants differently from conventionally bred varieties.

The reality is that the EU's GM regulations are outdated. While a precautionary component is still necessary in this regulatory framework, the Precautionary Principle overcompensates for the possible risks to a point that is no longer sensible for genome edited plants, let alone GMOs in general. Although GMOs are clearly defined from a legal perspective (see Section II), different risk assessments for GMOs and conventionally bred plants can no longer be justified. Because of the predominantly process-based ap-

\author{
42 USDA 2018 \\ 43 Belhaj et al. 2013; Ma et al. 2015; Qiwei et al. 2013; Schiml, Fauser and \\ Puchta 2016; Wook Woo et al. 2015 \\ $44 \quad$ Nicolia et al. 2014
}


proach, the EU treats genome edited products and other GM products differently from conventional products only because they are produced differently, and not because they are different. However, plant breeding methods, whether they are conventional or modern, are not good or bad per se. For example, the 1967 Lenape potato variety was produced using classical breeding methods and contained high levels of solanine in the potato tubers, causing sickness in consumers. ${ }^{45}$ As a consequence, new potato varieties are now regulated in some EU countries and their solanine levels must be tested. ${ }^{46}$

Thus, for a proper risk assessment of plants and products, a more product-based approach should be adopted in which the possible risks of the expressed traits are assessed regardless of the breeding method used. Perhaps a more product-based approach in the EU is inevitable, as genome edited products from, e.g., the USA are not regulated and will therefore not be labelled as genome edited products, yet are indistinguishable from conventional equivalents. This raises certain questions concerning current EU GM regulation: can it still be maintained if genome edited and conventional products are indistinguishable? Can genome edited products from exporting countries somehow be recognized by the EU (e.g., by obliging non-EU countries to label these products)? And can these products be refused, even as exporting countries increase their production of genome edited products? In other words, the regulation of genome edited products in non-EU countries will also impact the EU's GM regulation.

A novel principle named the Innovation Principle, proposed by the CEOs of major companies and promoted by the ERF (European Risk Forum) for awareness and adoption in 2013, should be the next step for the EU in regulating genome edited products. The Innovation Principle was proposed:

$\ldots$ to ensure that whenever policy or regulatory decisions are under consideration the impact on innovation as a driver for jobs and growth should be assessed and addressed. ${ }^{47}$

This does not mean that no precaution is involved. On the contrary, the ERF overview of the Innovation Principle states:

The Innovation Principle AND Precautionary Principle - The Innovation Principle is complementary with the Precautionary Principle, as

$45 \quad$ Patil et al. 1972

46 European Commission 2016

47 European Risk Forum (ERF) 2015 
precaution and innovation are equally important. The two principles should be used alongside each other, recognizing the need to protect society and the environment while also protecting Europe's ability to innovate. $^{48}$

In other words, the Innovation Principle is both precautionary and innovation-friendly. The EU's adoption of this principle would benefit genome editing technologies such as CRISPR/Cas9. This would be a significant step forward for the legislation of genome edited products. In the end, changing the EU's attitude towards CRISPR/Cas9 - and, ultimately, changing the EU's GM regulations - is not only important for making the EU less dependent on the import of palm oils, nor for saving rainforests: it is also important for the many future objectives that can be easily achieved using genome editing and other modern technologies. It is urgent that the extremely precautious EU switches to a method of product-based regulation, or at least adopts an innovation-friendly approach, before the EU economy unnecessarily falls behind others.

\section{Acknowledgements}

The COSMOS project has received funding from the European Union's Horizon 2020 research and innovation programme under grant agreement No 635405.

\section{References}

Adams, Keith L./Wendel, Jonathan F. (2005): Polyploidy and genome evolution in plants. Current Opinion in Plant Biology 8, 135-141.

Agence canadienne d'inspection des aliments: La biologie du Camelina sativa (L.) Crantz (caméline cultivée), 24/05/2017. Available at: http://www.inspection.gc.ca/vegetaux/vegetaux-a-caracteres-nouveaux/demandeurs/directive-9408/documents-sur-la-biologie/camelina-sativa-1/fra/1330971423348/1330971509470. (Accessed: 30/8/2017)

Andrée, Peter (2006): An Analysis of Efforts to Improve Genetically Modified Food Regulation in Canada. Science and Public Policy 33(5).

Belhaj, Khaoula/Chaparro-Garcia, Angela/Kamoun, Sophien/Nekrasov, Vladimir (2013): Plant genome editing made easy: targeted mutagenesis in model and crop plants using the CRISPR/Cas system. Plant Methods 9. 
Camara, Mark D./Ancell, Carrie A/Pigliucci, Massimo (2000): Induced mutations: A novel tool to study phenotypic integration and evolutionary constraints in Arabidopsis thaliana. Evolutionary Ecology Research 2, 1009-1029.

Canadian Food Inspection Agency (CFIA) (2007): Regulation of Agricultural Biotechnology in Canada: A Post-Secondary Educator's Resource. Available at: http://publications.gc.ca/collections/collection_2007/cfia-acia/A104-24-2007E.pdf.

Chikkali, Samir/Mecking, Stefan (2012): Refining of plant oils to chemicals by olefin metathesis. Angewandte Chemie - International Edition 51, 5802-5808.

Constable, A./Jonas, D./Cockburn, A./Davi, A./Edwards, G./Hepburn, P./HerouetGuicheney, C./Knowles, M./Moseley, B./Oberdö Rfer, R./Samuals, F. (2007): History of safe use as applied to the safety assessment of novel foods and foods derived from genetically modified organisms. Food and Chemical Toxicology 45, 2513 2525.

CORDIS: Camelina \& crambe Oil crops as Sources for Medium-chain Oils for Specialty oleochemicals. Available at: http://cordis.europa.eu/project/rcn/193330_en.html. (Version 14/07/2017; Accessed: 24/12/2017)

COSMOS: H2020 - COSMOS - Camelina \& crambe Oil crops as Sources for Mediumchain Oils for Specialty oleochemicals. Available at: http://cosmos-h2020.eu/. (Accessed: 24/12/2017)

Court of Justice of the European Union (2018): Organisms obtained by mutagenesis are GMOs and are, in principle, subject to the obligations laid down by the GMO Directive. Confédération paysanne and Others v Premier ministre and Ministre de l'Agriculture, de l'Agroalimentaire et de la Forêt, press release No 111/18. Available at: https://curia.europa.eu/jcms/upload/docs/application/pdf/2018-07/cp180111en.pdf.

Discover Life: map_of_Camelina_sativa.jpg $(720 \times 360)$. Available at: http://www.discoverlife.org/nh/maps/Plantae/Dicotyledoneae/Brassicaceae/Camelina/map_of_Camelina_sativa.jpg. (Accessed: 30/8/2017)

Dhuicq, Marie (2013): Plant-based Biofuels for Energy Supply for Small Villages. GENI (Global Energy Network Institute).

European Commission (2016): Summary Report of the Standing Committee on Plants, Animals, Food and Feed Held in Brussels on 23 June 2015 (Section Toxicological Safety of the Food Chain). Available at: https://ec.europa.eu/food/sites/food/files/safety/docs/reg-com_toxic_20150623_sum.pdf.

European Parliament (2001): Directive 2001/18/EC of the European Parliament and of the Council of 12 March 2001 on the deliberate release into the environment of genetically modified organisms and repealing Council Directive 90/220/EEC. Official Journal of the European Communities.

European Parliament (2003): Regulation (EC) No 1829/2003 of the European Parliament and of the Council of 22 September 2003 on genetically modified food and feed. Official Journal of the European Union.

European Risk Forum (ERF) (2015): The Innovation Principle - Overview. European Risk Forum. Available at: http://www.riskforum.eu/uploads/2/5/7/1/25710097/innovation_principle_one_pager_5_march_2015.pdf. 
FEDIOL (2017): Seeds production, imports, exports and crushing. FEDIOL 2016 Annual Statistics, 23/08/2017. Available at: http://www.fediol.be/data/1503559867Stat\%20seeds\%202016.pdf.

Feng, Zhengyan/Zhang, Botao/Ding, Wona/Liu, Xiaodong/Yang, Dong-Lei/Wei, Pengliang/Cao, Fengqiu/Zhu, Shihua/Zhang, Feng/Mao, Yanfei/Zhu, Jian-Kang (2013): Efficient genome editing in plants using a CRISPR/Cas system. Cell Research 23, 1229-1232.

Fitzherbert, Emily B./Struebig, Matthew J./Morel, Alexandra/Danielsen, Finn/Brühl, Carsten A./Donald, Paul F./Phalan, Ben (2008): How will oil palm expansion affect biodiversity? Trends in Ecology and Evolution 23, 538-545.

GeneCopoeia: Genome-CRISPTM CRISPR Products and Services - Complete Solutions for precision RNA-guided genome editing. Available at: http://www.genecopoeia.com/product/crispr-cas9/. (Accessed: 30/8/2017)

Government of Canada: Genetically Modified (GM) Foods and Other Novel Foods. Available at: https:/www.canada.ca/en/health-canada/services/food-nutrition/genetically-modified-foods-other-novel-foods.html. (Version 6/6/2018; Accessed: 26/8/2018)

Hartung, Frank/Schiemann, Joachim (2014): Precise plant breeding using new genome editing techniques: Opportunities, safety and regulation in the EU. Plant Journal 78, $742-752$.

Hunter, Philip (2014): 'Genetically Modified Lite' placates public but not activists. EMBO reports 15, 138-141.

ISAAA (1) (2017): Biotech Crop Highlights in 2016. Available at: http://www.isaaa.org/resources/publications/pocketk/foldable/Pocket\%20K16\%20(English)\%202017.pdf.

ISAAA (2) (2017): Global Status of Commercialized Biotech/GM Crops in 2017: Biotech Crop Adoption Surges as Economic Benefits Accumulate in 22 Years. ISAAA Brief 53. Available at: http://www.isaaa.org/resources/publications/briefs/53/download/isaaa-brief-53-2017.pdf.

Jiang, Wenzhi/Zhou, Huanbin/Bi, Honghao/Fromm, Michael/Yang, Bing/Weeks, Donald P. (2013): Demonstration of CRISPR/Cas9/sgRNA-mediated targeted gene modification in Arabidopsis, tobacco, sorghum and rice. Nucleic Acids Research 41, e188.

Jones, Huw D. (2015): Regulatory uncertainty over genome editing. Naute Plants 1.

Jouanin, Aurélie/Gilissen, Luud J.W.J./Boyd, Lesley A./Cockram, James/Leigh, Fiona J./Wallington, Emma J./Van den Broeck, Hetty C./Van der Meer, Ingrid M./Schaart, Jan G./Visser, Richard G.F./Smulders, Marinus J.M. (2017): Food processing and breeding strategies for coeliac-safe and healthy wheat products. Food Research International 110, 11-21.

Kalaitzandonakes, Nicholas/Alston, Julian M./Bradford, Kent J. (2007): Compliance costs for regulatory approval of new biotech crops. Nature Biotechnology 25, 509511.

Kriebel, David/Tickner, Joel/Epstein, Paul/Lemons, John/Levins, Richard/Loechler, Edward L./Quinn, Margaret/Rudel, Ruthann/Schettler, Ted/Stoto, Michael (2001): The 
Precautionary Principle in Environmental Science. Environmental Health Perspectives $109,871-876$.

Law Library of Congress: Restrictions on Genetically Modified Organisms: Canada. Available at: https://www.loc.gov/law/help/restrictions-on-gmos/canada.php. (Version 09/06/2015; Accessed: 26/8/2018)

Li, Jian-Feng/Norville, Julie E./Aach, John/McCormack, Matthew/Zhang, Dandan/Bush, Jenifer/Church, George M./Sheen, Jen (2013): Multiplex and homologous recombination-mediated genome editing in Arabidopsis and Nicotiana benthamiana using guide RNA and Cas9. Nature Biotechnology 31, 688-691.

Lightner, Jonathan/Caspar, Timothy (1998): Seed Mutagenesis of Arabidopsis. Methods in Molecular Biology 82, 91-103.

Ma, Xingliang/Zhang, Qunyu/Zhu, Qinlong/Liu, Wie/Chen, Yan/Qiu, Rong/Wang, Bin/Yang, ZhongfangLi, Heying/Lin, Yuru/Xie, Yongyao/Shen, Rongxin/Chen, Shuifu/Wang, Zhi/Chen, Yuanling/Guo, Jingxin/Chen, Letian/Zhao, Xiucai/Dong, Zhicheng/Liu, Yao-Guang/Y-g, Liu (2015): A Robust CRISPR/Cas9 System for Convenient, High-Efficiency Multiplex Genome Editing in Monocot and Dicot Plants. Molecular Plant 8, 1274-1284.

Mao, Yanfei/Zhang, Hui/Xu, Nanfei/Zhang, Botao/Gou, Feng/Zhu, Jian-Kang (2013): Application of the CRISPR-Cas system for efficient genome engineering in plants. Molecular Plant 6, 2008-2011.

MacLaughlin, Sara J. (2004): Food for the Twenty-First Century: An Analysis of Regulations for Genetically Engineered Food in the United States, Canada, and the European Union. Indiana International \& Comparative Law Review 14(1).

Maghuly, Fatemeh/Laimer, Margit (2017): Forward and Reverse Genetics for the Improvement of Jatropha. In: The Jatropha Genome. Compendium of Plant Genomes, 131-148. Springer, Cham. ISBN: 978-3-319-49653-5.

Merchant, Gary E./Stevens, Yvonne A. (2017): A new window of opportunity to reject process-based biotechnology regulation. GM Crops \& Food 6, 233-242.

Miao, Jin/Guo, Dongshu/Zhang, Jinzhe/Huang, Qingpei/Qin, Genji/Zhang, Xin/Wan, Jianmin/Gu, Hongya/Qu, Li-Jia (2013): Targeted mutagenesis in rice using CRISPRCas system. Cell Research 23, 1233-1236.

Montpetit, Eric (2005): A Policy Network Explanation of Biotechnology Policy Differences Between the United States and Canada. Journal of Public Policy 25(3), 339366.

Morineau, Céline/Bellec, Yannick/Tellier, Frédérique/Gissot, Lionel/Kelemen, Zsolt/Nogué, Fabien/Faure, Jean Denis (2012): Selective gene dosage by CRISPRCas9 genome editing in hexaploid Camelina sativa. Plant Biotechnology Journal 15, 729-739. Plant Physiology 135(2), 756-772.

Nature (2012): Method of the Year 2011. Nature Methods 9.

Nekrasov, Vladimir/Staskawicz, Brian/Weigel, Detlef/Jones, Jonathan D.G./Kamoun, Sophien (2013): Targeted mutagenesis in the model plant Nicotiana benthamiana using Cas9 RNA-guided endonuclease. Nature Biotechnology 31, 691-693. 
Nelson, David R/Schuler, Mary A./Paquette, Suzanne M./Werck-Reichhart, Daniele/Bak, Søren (2004): Comparative Genomics of Rice and Arabidopsis. Analysis of 727 Cytochrome P450 Genes and Pseudogenes from a Monocot and a Dicot.

Nicolia, Alessandro/Manzo, Alberto/Veronesi, Fabio/Rosellini, Daniele (2014): An overview of the last 10 years of genetically engineered crop safety research. Critical Reviews in Biotechnology 34, 77-88.

Osakabe, Yuriko/Watanabe, Takahito/Sugano, Shigeo S./Ueta, Risa/Ishihara, Ryosuke/Shinozaki, Kazuo/Osakabe, Keishi (2016): Optimization of CRISPR/Cas9 genome editing to modify abiotic stress responses in plants. Nature Scientific Reports 6.

Patil, B.C./Sharma, R.P./Salunkhe, D.K./Salunkhe, Kirti (1972): Evaluation of Solanine Toxicity. Food and Cosmetics Toxicology 10, 395-398.

Qiwei, Shan/Wang, Yanpeng/Li, Jun/Zhang, Yi/Chen, Kunling/Liang, Zhen/Zhang, Kang/Liu, Jinxing/Xi, Jianzhong Jeff/Qiu, Jin-Long/Gao, Caixia (2013): Targeted genome modification of crop plants using a CRISPR-Cas system. Nature 31, 686688.

Schaart, Jan G./van de Wiel, Clemens C.M./Lotz, Lambertus A.P./Smulders, Marinus J.M. (2016): Opportunities for Products of New Plant Breeding Techniques. Trends in Plant Science 21, 438-449.

Schiml, Simon/Fauser, Friedrich/Puchta, Holger (2016): CRISPR/Cas-Mediated SiteSpecific Mutagenesis in Arabidopsis thaliana Using Cas9 Nucleases and Paired Nickases. Methods in Molecular Biology 1469.

Upadhyay, Santosh K./Kumar, Jitesh/Alok, Anshu/Tuli, Rakesh (2013): RNA-Guided Genome Editing for Target Gene Mutations in Wheat. G3-Genes|Genomes|Genetics 3, 2233-2238.

USDA (2018): Secretary Perdue Issues USDA Statement on Plant Breeding Innovation. Press Release, Release No. 0070.18. Available at: https:/www.usda.gov/media/press-releases/2018/03/28/secretary-perdue-issues-usda-statement-plant-breeding-innovation

Wook Woo, Je/Kim, Jungeun/Il Kwon, Soon/Corval, Claudia/Woo Cho, Seung/Kim, Hyeran/Kim, Sang-Gyu/Kim, Sang-Tae/Choe, Sunghwa/Kim, Jin-Soo (2015): DNAfree genome editing in plants with preassembled CRISPR-Cas9 ribonucleoproteins. Nature Biotechnology 33.

World Commission on the Ethics of Scientific Knowledge and Technology (COMEST) (2005): The Precautionary Principle. United Nations Educational, Scientific and Cultural Organization. Available at: http://unesdoc.unesco.org/images/0013/001395/139578e.pdf.

Xie, Kabin/Yang, Yinong (2013): RNA-Guided Genome Editing in Plants Using a CRISPR-Cas System. Molecular Plant 6, 1975-1983.

Yang, Ning/Wang, Rongchen/Zhao, Yunde (2017): Revolutionize genetic studies and crop improvement with high-throughput and genome-scale CRISPR/Cas9 gene editing technology. Molecular Plant 10, 1141-1143.

Zhang, Feng/Wen, Yan/Guo, Xiong (2014): CRISPR/Cas9 for genome editing: progress, implications and challenges. Human Molecular Genetics 23, R40-R46. 
Zubr, Josef (1997): Oil-seed crop: Camelina sativa. Industrial Crops and Products 6, 113-119.

Zubr, Josef (2003): Dietary fatty acids and amino acids of Camelina sativa seed. Journal of Food Quality 26, 451-462. 